\title{
DOE PROGRESS REPORT 2008: Single-Molecule Methods for the Large-Scale Characterization of Expression Levels and Protein-Protein Interactions in Shewanella Oneidensis MR-1
}

\section{Summary:}

During the fourth year of this project, we have reached two major milestones:

1) We have completed a simplified proof of principle experiment using $T 7$ bacteriophage as a model system.

2) We have demonstrated targeting on the single molecule level of antibody conjugated QD to RNAP bound to genomic DNA.

The following sections provide some details on these achievements.

During the next year, we will concentrate on labeling flag sequences on DNA and combine these techniques to obtain nm-resolution localization of proteins on combed DNA with respect to known sequences in the DNA.

\section{1) Mapping promoters on the $T 7$ bacteriophage genome.}

We demonstrate the concept of mapping transcription factor binding sites with a proof-ofprinciple experiment aimed at locating a single QD-labeled protein species bound to multiple genomic targets. As a model biological system, we studied T7-RNA polymerase (RNAP) binding to promoters of the $\mathrm{T} 7$ bacteriophage genome. $\mathrm{T} 7$ is a virus that infects most Escherichia coli strains. It has a fully sequenced linear double-strand DNA genome containing 39,936 bp, with 17 identified promoter binding sites for T7 RNAP ${ }^{1}$.

To facilitate easy and selective binding of QDs to T7-RNAP, in vivo biotinylation was induced before RNAP purification. In vitro binding of the biotinylated-RNAP to the T7 genome was performed as described ${ }^{2}$. Since T7-RNAP binds weakly to DNA ${ }^{3}$, transcription was initiated with a nucleotide subset (GTP, UTP and CTP) to stabilize the complex. The sample was then labeled with the intercalating dye YOYO-1 and with streptavidin conjugated QDs.
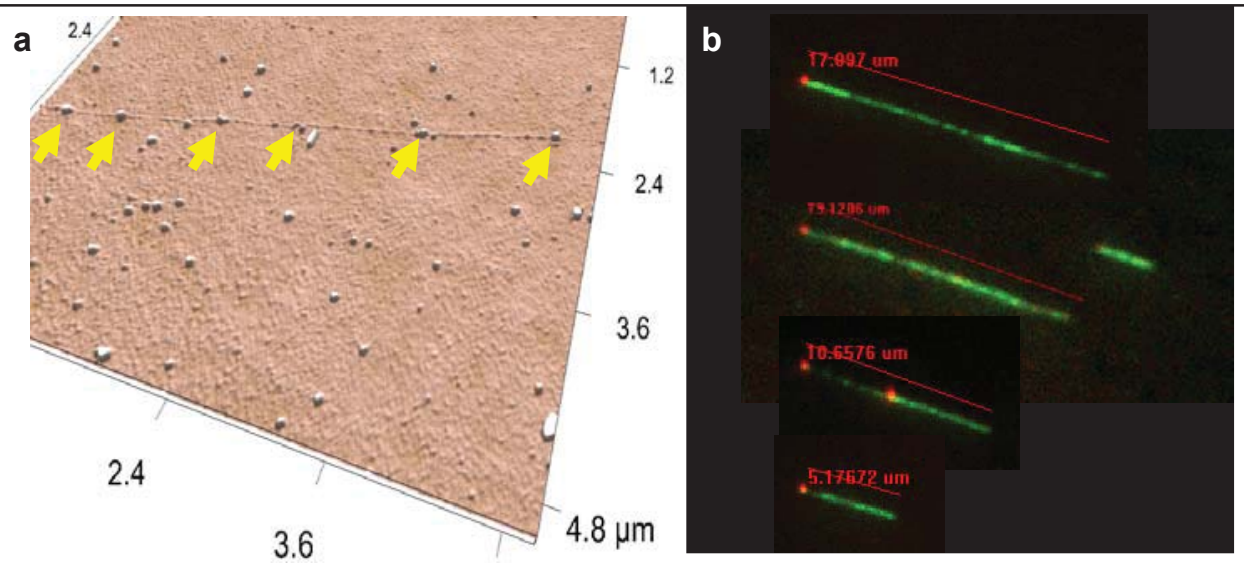

Figure1. Molecular combing of DNA-TF-QD complexes on polystyrene coated glass. a, 3D AFM image of Lambda-phage DNA with crosslinked RNAP. b, Cropped fluorescence images of T7-phage DNA (green) with crosslinked RNAP, tagged with qdot 655-streptavidin (red). 
We first aligned the sample by molecular combing, which reliably produces uniform stretching ${ }^{4}$. Combing of DNA with crosslinked RNAP using this method was successfully verified by AFM. However, when combing the QD-labeled complexes, we could only detect one QD at the end of the observed DNA molecules (Figure 1.).

We hypothesized that one of the bound QDs served to anchor the DNA to the surface, via nonspecific binding, and that the stretching force created by the receding meniscus was sufficient to detach the remaining T7-RNAP-QDs from the DNA ${ }^{5}$. To overcome these limitations, we utilized the flow-induced stretching technique ${ }^{6}$.

A $3 \mu \mathrm{L}$ droplet containing $\sim 5 \mathrm{ng}$ of DNA was deposited at the interface between two parallel coverslips, one of which was functionalized with polylysine. Capillary force immediately sucked the droplet in between the coverlips, stretching the DNA and immobilizing it on the polylysine surface, presumably by electrostatic interaction (Figure 2). We found that adding $0.1 \%$ of n-dodecyl- $\beta$-D-maltoside, a very mild nonionic surfactant ${ }^{7}$, improved the extension uniformity and reduced non-specific binding of free QDs to the polylysine surface ${ }^{8}$. The resulting fluorescence images clearly show individual QD fluorescent spots on many of the DNA molecules (Figure 2).

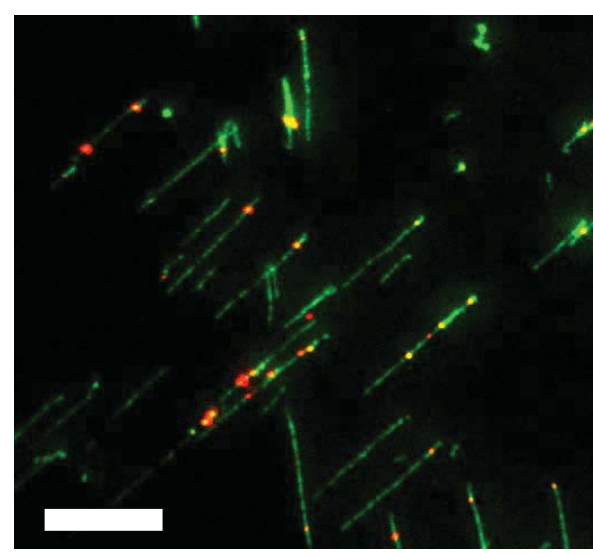

Figure 2. Typical $50 \mu \mathrm{m}^{2}$ field of view for the T7-phage sample described in the text. overlaid green and red images acquired with an EMCCD (DU897, Andor). (Scale bar $10 \mu \mathrm{m})$.

The small number of bound QDs per DNA molecule was in part due to the sub-stoichiometric amount of QDs used in the experiment to reduce non-specific binding of QDs on the surface. AFM imaging of the sample indicates that most promoter sites were however occupied by T7RNAP (Figure 3). DNA fibers decorated with protrusions of two typical sizes were observed. We attributed smaller protrusions to bare T7-RNAP and the larger protrusions to T7-RNAPQD.

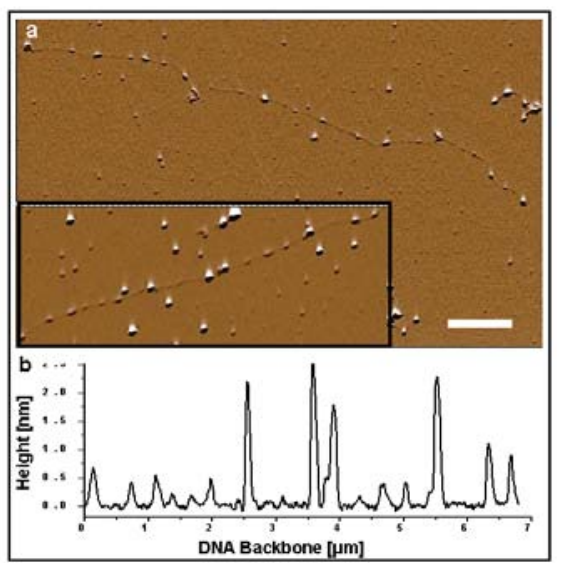

Figure 3. Evaluation of RNAP and QD binding on the T7-phage genome. a, Amplitude AFM scans of the T7DNA-RNAP-QD complex, before (inset) and after heparin treatment. Protrusions of two typical sizes, corresponding to the RNAP $(0.5 \mathrm{~nm})$ and RNAP-QD $(>1.5 \mathrm{~nm})$ are visible on the DNA backbone. (Scale-bar $1 \mu \mathrm{m}$ ). $\mathbf{b}$, Cross section along the DNA located in the inset, depicting the height of the various protrusions. 
We first evaluated the uniformity and degree of extension by measuring the length of QDlabeled DNA molecules. We found that DNA was extended to $86 \pm 6 \%$ of its B-DNA contour length $(3.42 \mathrm{bp} / \mathrm{nm})$. This dispersion of the extension factor is the largest source of uncertainty for absolute distance measurements. Distances between the DNA end and QDs were measured manually using a custom code. To refine the analysis, we first corrected for variations in extension factor from molecule to molecule by dividing each distance by the total length of the individual DNA molecule. Since this sample did not contain any marker to indicate DNA orientation, QDs are matched to known promoters by comparing their measured positions to theoretical ones and choosing the orientation resulting in the best global match. The position histogram in Figure 4 shows the frequency of QD positions along the genome relative to the positions of known RNAP promoters. Representative genome images, scaled to span the full promoter map are presented, and QD labels may be traced to their matching promoters. The distribution is clearly discrete, with a considerable degree of correlation between promoters and QD clusters. This distribution indicates that QD positions are not random, and represent mostly specific binding events of RNAP under our experimental conditions.

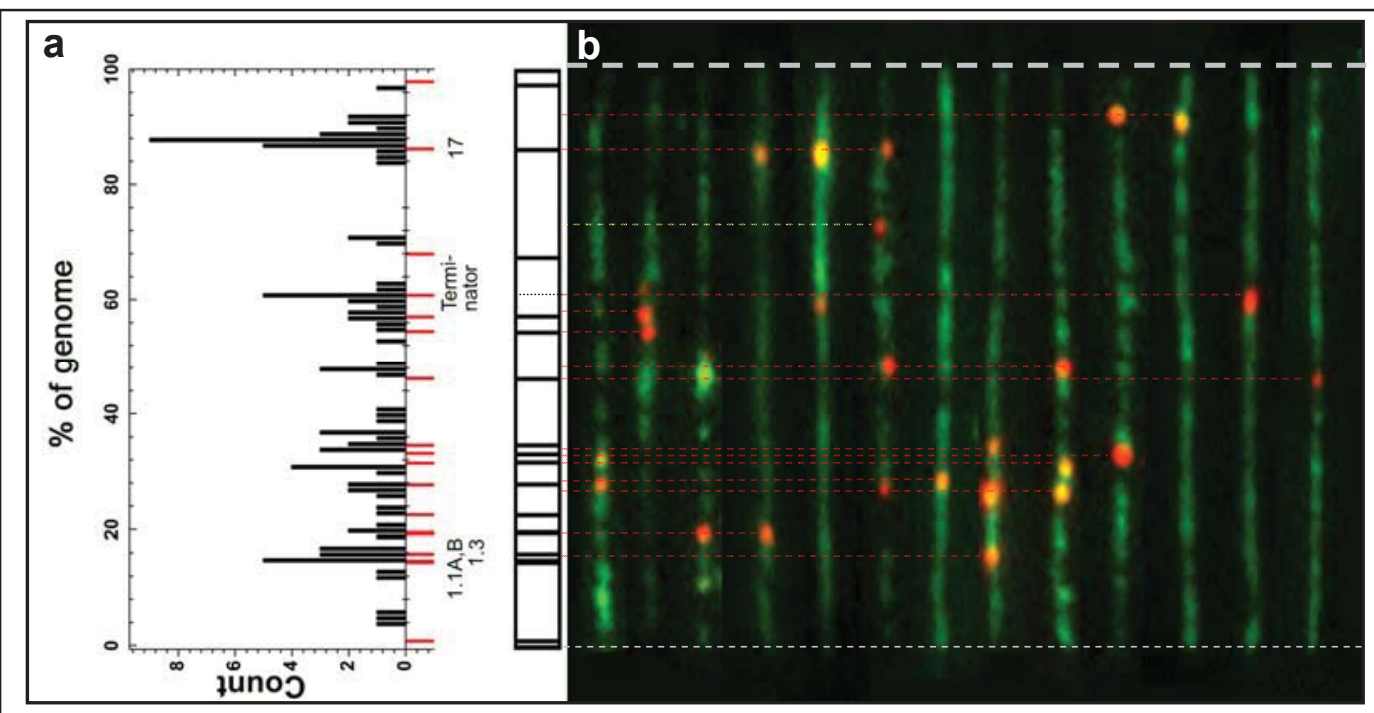

Figure 4. Evaluation of promoter occupancy. a, Known promoter positions (red) and histogram constructed from 70 genomes having two identifiable ends (black). Data is presented in normalized percentage units going from left to right in the direction of transcription. The distance between a bound QD and the DNA end was normalized to units of $\%$ of the total genome by taking the ratio of the distance and the total length. Orientation was determined by assigning one QD per DNA to the best matching promoter (bin $=1 \%$, [400bp]). b, Cropped fluorescence images of full length T7-phage genomes, scaled to span the full promoter map. White dashed lines indicate the DNA extremities, red and yellow dashed line are guides for the eye and connect QD binding locations on different molecules to the promoter map.

Most notable is the high occupancy of the region corresponding to promoter 17 located at $86 \%$ of the full genome length as well as that of a known strong terminator at $61 \%$. A numerical simulation of our experiment showed good agreement with our observation, provided we assigned a binding probability for this promoter twice as large as that of other consensus promoters (Supplementary Figure 4). We could find no reported explanation for this higher occupancy of promoter 17 relative to other regions, suggesting that this 
observation could be of biological importance. This simple experiment thus indicates that our method may yield insightful results even in relatively well known systems such as T7.

\section{2) Targeting single transcription factors by antibody conjugated QDs}

We have recently been able to optimize a conjugation reaction enabling antibody conjugation to peptide coated QDs. These QDs were tested on the single molecule level by targeting E. coli RNAP bound to specific promoters on the T7 genome. Binding of RNAP to the expected loci on the genome was first confirmed by AFM and then followed by fluorescence imaging of the sample. The detected QD is visible at the expected location, confirming specific binding of the conjugated QD.

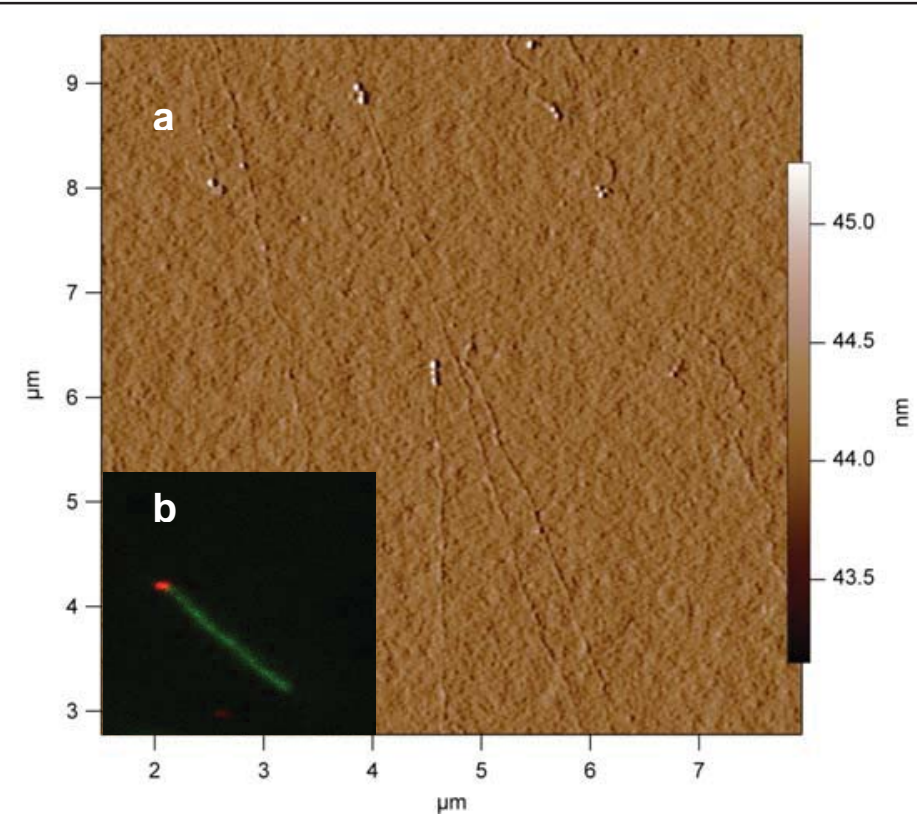

Figure 5. Targeting RNAP with antibody conjugated QDs. a, The T7 Bacteriophage genome was reacted with Ecoli RNAP enzyme which has 4 specific binding sites at one of the genome's ends. Atomic force microscope imaging of the complex shows 2-4 bumps at the end of the DNA fiber, confirming binding of RNAP to the expected location.b, QDs were peptide coated and conjugated to Anti-RNAP. These QDs (red) were reacted with the DNA-RNAP complex, the DNA was stained with YOYO-1 (green) and deposited on glass. Fluorescence imaging confirms specific QD binding on the single molecule level

\section{Publications}

1. Yuval Ebenstein, Natalie Gassman, Josh Antelman, Younggyu Kim, Sam Ho1, Soohong Kim, Robin Samuel, Xavier Michalet and Shimon Weiss (2008) Lighting up individual transcription factors with quantum dots (Submitted)

\section{References}

1. Dunn, J.J. \& Studier, F.W. Complete nucleotide sequence of bacteriophage T7 DNA and the locations of T7 genetic elements. Journal of molecular biology 166, 477-535 (1983). 
2. Gueroui, Z., Place, C., Freyssingeas, E. \& Berge, B. Observation by fluorescence microscopy of transcription on single combed DNA. Proceedings of the National Academy of Sciences 99, 6005-6010 (2002).

3. Ikeda, R.A. \& Richardson, C.C. Interactions of the RNA polymerase of bacteriophage T7 with its promoter during binding and initiation of transcription. Proceedings of the National Academy of Sciences 83, 3614-3618 (1986).

4. Allemand, J.F., Bensimon, D., Jullien, L., Bensimon, A. \& Croquette, V. pHdependent specific binding and combing of DNA. Biophysical journal 73, 2064-2070 (1997).

5. Bensimon, D., Simon, A.J., Croquette, V. \& Bensimon, A. Stretching DNA with a Receding Meniscus: Experiments and Models. Physical Review Letters 74, 4754 (1995).

6. Meng, X., Benson, K., Chada, K., Huff, E.J. \& Schwartz, D.C. Optical mapping of lambda bacteriophage clones using restriction endonucleases. Nature Genetics 9, 432 438 (1995).

7. Stubbs, G.W., Smith, H.G. \& Litman, B.J. Alkyl glucosides as effective solubilizing agents for bovine rhodopsin. A comparison with several commonly used detergents. Biochimica et Biophysica Acta (BBA) - Biomembranes 426, 46-56 (1976).

8. Huang, B., Wu, H., Kim, S. \& Zare, R.N. Coating of poly(dimethylsiloxane) with ndodecyl-[small beta]-d-maltoside to minimize nonspecific protein adsorption. Lab on a Chip 5, 1005-1007 (2005). 